\title{
Anthocyanin biosynthesis regulation in colored seed coat of Vigna unguiculata (L.) Walp.
}

\author{
Krylova E. ${ }^{1 *}$, Mikhailova A. ${ }^{1,2}$, Khlestkina E. ${ }^{1}$ \\ ${ }^{1}$ Federal Research Center the N.I. Vavilov All-Russian Institute of Plant Genetic Resources (VIR), \\ Saint Petersburg, Russia \\ ${ }^{2}$ Saint Petersburg State University, Saint Petersburg, Russia \\ *e.krylova@vir.nw.ru
}

Key words: Vigna unguiculata, cowpea, VIR collections, anthocyanins, MBW complex, marker-assisted selection

Motivation and Aim: Healthy lifestyle and a correctly balance diet constitute one of the main trends in development of the modern society. Colored antioxidant-rich products due to its high flavonoids content reduce the risks of oncological, cardiovascular and age-related neurodegenerative diseases. Such a product may be colored seeds which pattern is one of important feature for consumers. So that the colored seed coats of cowpea (Vigna unguiculata (L.) Walp.) from VIR collection play a potential important role as basic dietetic products for millions of people. Nowadays very little is known about regulatory MBW complex ( $R 2 R 3-M y b, b H L H-M y c$ and $W D R$ genes) involved in anthocyanin biosynthesis in colored cowpea seed coats.

Methods and Algorithms: The structural organization of $M y b$ was established with help in silico analysis using LIS (Legume Information System, https://legumeinfo.org) and Pfam databases. We used the chloroform-isoamyl alcohol extraction protocol to isolate genomic DNA from the cowpea young seedlings of accessions that have various seed coat color (white, beige, purple and black). PCR with the $M y b$-specific gene primers was conducted to reveal the gene deletion sites among all investigated samples. PCR amplification products were size-separated by horizontal electrophoresis in $1 \%$ agarose gels. The PCR products were excised and purified for further Sanger sequencing. The resequencing results were analyzed using UGENE software.

Results: In silico analysis revealed that $M y b$ gene has two conservative R2 and R3 functional domains, which are typical for $R 2 R 3-M y b$ gene family. The $M y b$ gene was conservative among all studied accessions with the anthocyanin-rich black seed coat accessions. At the same time only for some non-anthocyanin seed coat samples (white, beige and purple) the PCR-products were observed. During the resequencing data analysis we detected two nucleotide insertion in intron for the purple seed coat accession. In addition, the two SNPs in untranslated region for beige and purple colored seed coat samples were detected. The minor differences of $M y b$ gene sequences point out at conservative character of $M y b$ among all investigated samples. The non-anthocyanin phenotype can be result of possible mutations in gene sequence coding the bHLH transcription factor.

Conclusion: We propose the complementary interaction of $M y b$ and $b H L H$ genes in formation of anthocyanin-rich black seed coat in cowpea. These achievements can be useful for further marker-assisted selection of cowpea varieties with the high dietary value. 